\title{
The Performance of the Modified Subgradient Algorithm on Solving the 0-1 Quadratic Knapsack Problem
}

\author{
Aydin SIPAHIOGLU, Tugba SARAÇ \\ Industrial Engineering Department of Eskisehir Osmangazi University \\ Meselik 26480, Eskisehir, Turkey \\ e-mail:asipahi@ogu.edu.tr; tsarac@ogu.edu.tr
}

Received: August 2008; accepted: May 2009

\begin{abstract}
In this study, the performance of the modified subgradient algorithm (MSG) to solve the $0-1$ quadratic knapsack problem (QKP) was examined. The MSG was proposed by Gasimov for solving dual problems constructed with respect to sharp Augmented Lagrangian function. The MSG has some important proven properties. For example, it is convergent, and it guarantees zero duality gap for the problems such that its objective and constraint functions are all Lipschtz. Additionally, the MSG has been successfully used for solving non-convex continuous and some combinatorial problems with equality constraints since it was first proposed. In this study, the MSG was used to solve the QKP which has an inequality constraint. The first step in solving the problem was converting zero-one nonlinear QKP problem into continuous nonlinear problem by adding only one constraint and not adding any new variables. Second, in order to solve the continuous QKP, dual problem with "zero duality gap" was constructed by using the sharp Augmented Lagrangian function. Finally, the MSG was used to solve the dual problem, by considering the equality constraint in the computation of the norm. To compare the performance of the MSG with some other methods, some test instances from the relevant literature were solved both by using the MSG and by using three different MINLP solvers of GAMS software. The results obtained were presented and discussed.
\end{abstract}

Keywords: quadratic knapsack problem, sharp augmented lagrangian function, MSG, integer programming.

\section{Introduction}

The knapsack problem (KP) is a well-known combinatorial optimization problem. The classical KP seeks to select, from a finite set of items, the subset, which maximizes a linear function of the items chosen, subject to a single inequality constraint. In many real life applications it is important that the profit of a packing also should reflect how well the given items fit together. One formulation of such interdependence is the quadratic knapsack problem. The quadratic knapsack problems (QKP) ask to maximize a quadratic objective function subject to a single capacity constraint. Some application areas of the QKP are determination of the optimal sites for communication satellite earth stations with 
a budget constraint, and similarly the determination of the location of railway stations and freight handling terminals and airports.

QKP was introduced in 1980 by Gallo, Hammer and Simeone, who also presented the first branch-and-bound algorithm using the bounds based on upper planes. In 1986, a branch and bound algorithm for QKP was proposed by Chaillou, Hansen and Mahieu. In their proposal, the computation of an upper bound was based on Lagrangean relaxation. Then, two upper bounds based on Lagrangean decomposition were presented for QKP by Michelon and Veuilleux (1996). In the same year, Billionnet and Calmels presented a branch-and-cut approach for QKP. An exact algorithm was developed for QKP by Caprara, Pisinger and Toth in 1999. In 2000, Helmberg, Rendl and Weismantel proposed a number of upper bounds for QKP based on semi definite programming. In 2003, Billionnet and Soutif introduced an exact method based on computation of an upper bound by means of Lagrangean decomposition. This method allows for finding the optimum of instances with up to 150 variables whatever their densities are, and with up to 300 variables for medium and low densities. In 2005, Julstrom proposed a greedy genetic algorithm (GA) whose operators implement the strategies of the two QKP greedy heuristics. In Julstrom's study, by using the greedy GA, near optimal solutions were obtained with very small error for the test instances with 100 and 200 variables in a reasonable short time.

In this study, the performance of the modified subgradient algorithm (MSG) to solve the $0-1$ quadratic knapsack problem (QKP) was examined. Then the results obtained this way were compared with the results obtained when QKP was regarded as a quadratic integer model and three different GAMS MINLP solvers (DICOPT, SBB, and BARON) were used.

The MSG algorithm was proposed by Gasimov in 2002 for solving a continuous nonlinear model with respect to sharp augmented Lagrangean function. In order to use the MSG algorithm, the QKP model should be converted into the continuous form first. In this study, Li's procedure $(\mathrm{Li}, 1992)$ was used due to the fact that this procedure adds only one new constraint to the model, and it does not require adding new variables. After converting, the dual problem with "zero duality gap" should be obtained and solved by using the augmented Lagrangean function. Different methods or solvers can also be used in solving the problem. In this study, CONOPT and MINOS solvers of the GAMS software were used for solving the dual model.

In the steps of the MSG algorithm, two different formulations were given for step size parameter by Gasimov and Ustun (2007). Indeed one of them may be used. However, in order to determine differences between these two step parameters, in this study, both of them were used on the same literature test instances and results were compared. All test problems were taken from Soutif's QKP page in University of Paris web site. They were solved by using DICOPT, SBB, BARON solvers of GAMS software and by using the MSG with two different step size parameters separately. As a result, it was shown that the MSG is a promising and competitive algorithm to solve QKP.

The organization of this paper is as follows. In section two, the modified subgradient algorithm is introduced. In section three, solving QKP procedure by using the MSG 
algorithm is explained. Computational results are reported in section four and finally, conclusions are given in the fifth section.

\section{Modified Subgradient Algorithm}

One of the classes of the exact methods used for solving $0-1$ integer problems with nonconvex objective and/or constraints is based on penalty function approaches. The simple examples show that in many cases the solution to the penalty problem can be made sufficiently close to the optimal solution of the original problem by choosing the penalty parameter large enough. However, solving a penalty problem with a very large penalty parameter leads to computational difficulties (see, for example, Bazaraa et al., 2006, and Bertsekas, 1995). Ordinary Lagrangian duality underlies many efficient algorithms for convex minimization problems. A key ingredient is the strong duality. Lagrangian relaxation and decomposition methods have been extensively used for solving linear integer problems (see, Michelon and Maculan, 1993; Michelon and Veuilleux, 1996). Unfortunately, ordinary Lagrangian methods often end up with a duality gap and fail to identify an optimal solution of the primal integer optimization problems such as the quadratic $0-1$ problems which are non-convex, in general (Li, 1999).

In recent years, different augmented Lagrangian duality schemes which are able to eliminate duality gap in a wide class of non-convex optimization problems and to provide solution algorithms, have been extensively studied (see, Gasimov, 2002; Gasimov and Rubinov, 2004; Burachik et al., 2006; Gasimov and Ustun, 2007; Burachik and Kaya, 2007; Burachik et al., in press). The MSG algorithm was proposed by Gasimov in 2002, and then Gasimov and Rubinov introduced a general version of this algorithm by modifying it for generalized augmented Lagrangian dual problems and extended the circle of problems solvable by this method in 2004. Gasimov and Ustun (2005) examined the MSG algorithm by applying it to solve the non-convex zero-one quadratic assignment problems. Burachik et al. (2006) gave a new convergence analysis for the MSG algorithm and they proposed new formulas for the step-size parameters. In 2007, Gasimov and Ustun proposed a generalized version of the MSG algorithm to solve sharp augmented Lagrangian dual problems. Burachik et al. (in press) proposed an inexact version of the MSG algorithm which may allow for solving problems with less computational effort.

The MSG algorithm can solve the non-convex optimization problems with equality constraint. If the problem is not convex, using the classical Lagrangean may lead to nonzero duality gap. However, if the sharp augmented Lagrangean function is used; this problem can be eliminated for a large scale of problems. It is proven that when the objective and constraint functions are all Lipschitz then the sharp augmented Lagrangean guaranties the zero duality gap (Gasimov, 2002). This is a very important property but it is not the only one because the MSG algorithm has other outstanding properties, too. For example, the MSG algorithm converges to the optimal dual value when the step-size is conveniently chosen. It does not require convexity or differentiability conditions on 
the primal problem and it does not use any penalty parameter. In other words, the MSG algorithm can find the optimal value if the step-size parameter and other parameters are properly chosen. That is the reason why the MSG algorithm is preferable. However, at the end of the solution process, the obtained solution value may not be optimal. Success of the MSG depends on success of the solver used for solving dual model. If solver guarantees the optimal solution of the dual model, the MSG can find the optimal value on condition using proper parameter values. Otherwise, it can not be said anything about optimality of the obtained solution. Nevertheless, it is observed that the MSG algorithm can solve the problems in reasonable short times. For example, 32b, the hardest test problem of Quadratic Assignment Problem (QAP), was solved in $4.32 \mathrm{~s}$ by using the MSG algorithm, although the other techniques need 7 years to solve. (see Gasimov and Ustun, 2005; Burachik et al., 2006; Gasimov and Ustun, 2007). The MSG algorithm is explained shortly as follows:

Let the primal problem $P$ be given as follows,

$$
\begin{aligned}
& \min P=\min _{x \in S} f(x), \\
& \text { subject to } g(x)=0,
\end{aligned}
$$

where $S$ is a compact subset of a metric space $X$, and $f: X \rightarrow R$ and $g: X \rightarrow R^{n}$ are given functions. The sharp augmented Lagrangean function $L: S \times R^{n} \times R_{+} \rightarrow R$ associated with $P$ :

$$
L(x, u, c)=f(x)+c\|g(x)\|-\langle g(x), u\rangle
$$

where $c$ and $u$ are the dual variables, $\|\cdot\|$ is the Euclidean norm and $\langle\cdot, \cdot\rangle$ is the Euclidean inner product on $R^{n}$.

The dual function $H: R^{n} \times R_{+} \rightarrow R$ associated with the problem $P$ is defined as

$$
H(u, c)=\min _{x \in S} L(x, u, c), \quad \text { for } u \in R^{n}, \text { and } c \in R_{+} .
$$

Then the dual problem $P^{*}$ is given by:

$$
\max _{(u, c) \in R^{n} \times R_{+}} H(u, c) .
$$

The steps of the MSG algorithm given by Gasimov and Ustun (2005) are as follows.

Initialization step: Choose a vector $\left(u_{1}, c_{1}\right) \in R^{n} \times R_{+}$. Let $k=1$.

Step 1. Given Lagrange multipliers $\left(u_{k}, c_{k}\right)$, solve the following sub problem:

$$
\begin{aligned}
& \underset{x \in S}{\operatorname{Minimize}} f(x)+c c_{k}\|g(x)\|-\left\langle u_{k}, g(x)\right\rangle, \\
& \text { subject to } f(x)+c c_{k}\|g(x)\|-\left\langle u_{k}, g(x)\right\rangle \leqslant \bar{H} .
\end{aligned}
$$


Let $x_{k}$ be the global solution of this problem. If $\left\|g\left(x_{k}\right)\right\|=0$ then stop. $\left(u_{k}, c_{k}\right)$ is a solution to the dual problem $\left(P^{*}\right), x_{k}$ is a solution to $(P)$. Otherwise, go to Step 2.

Step 2. Let

$$
u_{k+1}=u_{k}-s_{k} g\left(x_{k}\right), c_{k+1}=c_{k}+\left(s_{k+} \varepsilon_{k}\right)\left\|g\left(x_{k}\right)\right\|,
$$

where $s_{k}$ and $\varepsilon_{k}$ are positive scalar step sizes, replace $k$ by $k+1$ and repeat Step 1 .

In practice, following step size formulation can be used:

$$
s_{k}=\frac{\delta\left(\bar{H}-L\left(x_{k}, u_{k}, c_{k}\right)\right)}{5\left\|g\left(x_{k}\right)\right\|^{2}},
$$

where, $\bar{H}$ is an approximate optimal value or an upper bound for the dual problem and $0<\delta<2$.

Choosing a value for the upper bound $\bar{H}$ is crucial for the performance of the MSG algorithm. In practice such a value may be taken by different ways. It is very easy to find an upper bound for the QKP as the original objective function of the QKP is maximization. After converting into minimization form, zero will be the upper bound for all the converted QKPs because the values of the objective functions are always negative. The running times for the problems are not so long. Therefore, trying the different upper bounds is not hard and the best upper bound can be found in a reasonable time. Another important issue is calculating the $\varepsilon_{k}$ value. An interval is given for $\varepsilon_{k}$ by Gasimov (2002).

Theorem 1. Let $\left\{\left(u_{k}, c_{k}\right)\right\}$ be the sequence of dual variables generated by the MSG algorithm. Assume that $\left(u_{k}, c_{k}\right)$ is not a solution of the dual problem for any $k$, that is, $g\left(x_{k}\right) \neq 0$ for all $k$. Then

(a) Assume that there exists a dual solution. If

$$
0<\varepsilon_{k}<s_{k}=\frac{\alpha\left(\bar{H}-L\left(x_{k}, u_{k}, c_{k}\right)\right)}{5\left\|g\left(x_{k}\right)\right\|^{2}},
$$

then $d_{k+1}-d_{k}<0$, where $d_{k}=d\left((\bar{u}, \bar{c}),\left(u_{k}, c_{k}\right)\right)$ is distance between the optimal dual solution and the pair of dual variables calculated at the kth iteration of the algorithm and $0<\delta<2$.

(b) Assume again that that there exists a dual solution and that $f$ and $g$ are continuous, $S$ is compact, and a feasible solution exists. If

$$
0<\varepsilon_{k}<s_{k}=\frac{\left(\bar{H}-L\left(x_{k}, u_{k}, c_{k}\right)\right)}{5\left\|g\left(x_{k}\right)\right\|^{2}}
$$

then $L\left(x_{k}, u_{k}, c_{k}\right) \rightarrow \bar{H}$.

Since the objective and constraint functions of the problem continuous nonlinear problem (CNP) given in (10)-(12) are all continuous and the set $S$ for the problem (CNP) is compact, it guarantees the duality gap property and the existence of solutions to $(P)$. 
Theorem 2 was given by Burachik et al. (2006) and Theorem 3 and its proof were also given by Gasimov (2002) to show that if $x_{k}$ is a solution to $(P)$ and $(\bar{u}, \bar{c})$ is a solution to $\left(P^{*}\right)$ if and only if $g\left(x_{k}\right)=0$.

Theorem 2. Suppose that $f(x)$ and $g(x)$ are continuous, $S$ is compact, and a feasible solution of $(P)$ exists. Then inf $P=\sup P^{*}$ and $S(P) \neq \varnothing$. Furthermore, the dual function $H$ is concave and finite everywhere on $R^{n} \times R_{+}$. Consequently, this maximization problem is effectively unconstrained.

Theorem 3. Let Inf $P=S u b P^{*}$ and for some $(\bar{u}, \bar{c}) \in Y^{*} \times R_{+}$,

$$
\min _{x \in S} L(x, \bar{u}, \bar{c})=f(\bar{x})+\bar{c}\|g(\bar{x})\|-\langle g(\bar{x}), \bar{u}\rangle .
$$

Then $\bar{x}$ is a solution to $(P)$ and $(\bar{u}, \bar{c})$ is a solution to $\left(P^{*}\right)$ if and only if

$$
g(x)=0
$$

where $X$ is any topological linear space, $S \subset X$ is a certain subset of $X, Y$ be a real normed space and $Y^{*}$ is its dual.

The following step-size formulation was proposed and proven that it forces convergence of the dual values towards the optimal value $\bar{H}$ by Burachik et al. (2006).

$$
S_{k}=\delta \frac{\left(\left(\bar{H}-L\left(x_{k}, u_{k}, c_{k}\right)\right)+\alpha\left(\bar{c}-c_{k}\right)\left\|g\left(x_{k}\right)\right\|\right)}{\left(1+(1+\alpha)^{2}\right)\left\|g\left(x_{k}\right)\right\|^{2}}, \quad 0<\delta<2
$$

In 2007, Gasimov and Ustun proposed new formulations (3), (4) and (5) instead of (1) and (2). These formulations are given below:

$$
\begin{aligned}
& u_{k+1}=u_{k}-\alpha s_{k} g\left(x_{k}\right), c_{k+1}=c_{k}+(1+\alpha) s_{k}\left\|g\left(x_{k}\right)\right\|, \\
& S_{k}^{1}=\frac{\delta\left(\alpha\left(\bar{H}-L\left(x_{k}, u_{k}, c_{k}\right)\right)+\left(\bar{c}-c_{k}\right)\left\|g\left(x_{k}\right)\right\|\right)}{\left(\alpha^{2}+(1+\alpha)^{2}\right)\left\|g\left(x_{k}\right)\right\|^{2}}, \\
& S_{k}^{2}=\frac{\delta \alpha\left(\bar{H}-L\left(x_{k}, u_{k}, c_{k}\right)\right)}{\left(\alpha^{2}+(1+\alpha)^{2}\right)\left\|g\left(x_{k}\right)\right\|^{2}}
\end{aligned}
$$

where $\alpha>0$ and $0<\delta<2$.

In this study, both of them were used separately in order to determine the differences between them. In these formulations, $c$ is not a penalty parameter. It is a dual variable and its value is calculated as a function of the step size parameter. 


\section{Solving the QKP by Using the MSG Algorithm}

The QKP is a well known combinatorial optimization problem in Operational Research literature and it is defined as follows:

$$
\begin{aligned}
& \max z=\sum_{i=1}^{n} p_{i} x_{i}+\sum_{i=1}^{n-1} \sum_{j=i+1}^{n} p_{i j} x_{i} x_{j}, \\
& \text { subject to } \sum_{i=1}^{n} w_{i} x_{i} \leqslant a, \\
& x_{i}, x_{j} \in\{0,1\}, \quad i, j=1, \ldots, n,
\end{aligned}
$$

where $n$ items to pack in some knapsack of capacity $a$. Each item $i$ has a weight $w_{i}$ and profit $p_{i}$ which is the profit achieved, if item $i$ is selected. $p_{i j}$ is the profit achieved if both items $i$ and $j$ are selected and the objective is maximizing the profit sum of the included items without having the weight sum to exceed $a$. All the model parameters $p_{i}, p_{i j}, w_{i}$ and $a$ are positive numbers. In addition, the parameter $a$ should satisfy the following condition, $\max _{i} w_{i} \leqslant a \leqslant \sum_{i=1}^{n} w_{i}$.

In order to solve the QKP by using the MSG algorithm, QKP should be converted into the continuous form first. In this study, $0-1$ quadratic knapsack problem $(P)$ was converted into continuous nonlinear problem (CNP) by adding the only one constraint which was proposed by $\mathrm{Li}$ in 1992.

$$
\sum_{i=1}^{n}\left(x_{i}-x_{i}^{2}\right)=0, \quad \text { with } 0 \leqslant x_{i} \leqslant 1
$$

Capacity constraint was reduced to the equality constraint by adding a slack variable and the maximization problem was converted to the minimization one

$$
\max (f(x))=-\min (-f(x))
$$

The equivalent formulation of $(P)$ as a continuous nonlinear problem with equality constraints $(\mathrm{CNP})$ can be written as follows:

$$
\begin{aligned}
& (\mathrm{CNP}) \quad \min z=-\sum_{i=1}^{n} p_{i} x_{i}-\sum_{i=1}^{n-1} \sum_{j=i+1}^{n} p_{i j} x_{i} x_{j}, \\
& \text { subject to } \sum_{i=1}^{n} w_{i} x_{i}+\text { slack }-a=0, \\
& \sum_{i=1}^{n}\left(x_{i}-x_{i}^{2}\right)=0, \\
& 0 \leqslant x_{i} \leqslant 1, \quad i=1, \ldots, n, \text { slack } \geqslant 0 .
\end{aligned}
$$


Then Augmented Lagrangean Function $L(u, c, x)$ for $(\mathrm{CNP})$ is constructed as given below.

$$
\begin{aligned}
L(u, c, x)= & \sum_{i=1}^{n} p_{i} x_{i}+\sum_{i=1}^{n-1} \sum_{j=i+1}^{n} p_{i j} x_{i} x_{j} \\
& +c \sqrt{\left(\sum_{i=1}^{n} w_{i} x_{i}+\text { slack }-a\right)^{2}+\left(\sum_{i=1}^{n}\left(x_{i}-x_{i}^{2}\right)\right)^{2}} \\
- & u_{1}\left(\sum_{i=1}^{n} w_{i} x_{i}+\text { slack }-a\right)-u_{2}\left(\sum_{i=1}^{n}\left(x_{i}-x_{i}^{2}\right)\right), \\
& 1 \leqslant x_{i} \leqslant 0, i=1, \ldots, n, \text { slack } \geqslant 0 .
\end{aligned}
$$

The last step of the algorithm is solving this sub-problem. Any method or solver can be used to do this. In this study, CONOPT solver with step parameter $s_{k}^{1}$ and MINOS solver with step parameter $s_{k}^{2}$ were used separately. Computational results are given in Section 4.

\section{Computational Results}

In this section, the solving results of twenty QKP instances from the pertinent literature are presented. All instances were solved by using three different GAMS MINLP solvers (DICOPT, SBB and BARON) as integer QKP model and by using the MSG algorithm with two different step size parameters $s_{k}^{1}$ and $s_{k}^{2}$. All computational experiments were conducted on HP6000 workstation and their computational time and the objective function values were reported and compared.

The first ten instances were called as Group 1 and others were called as Group2. In order to define structure of test instances, number of available objects $(n)$ and density percentage of non-zero $p_{i j}-(d)$ values were used. The test instances in Group 1 have 100 objects with 0.25 densities, and the test instances in Group 2 have 200 objects with 1 density. It is known that solving a problem of high density is relatively harder. On the other hand, the optimal solutions were known for these test instances due to the fact that all instances were solved to optimality by Billionet and Soutif (2003) and the relevant web site ${ }^{1}$ reports their optimum values.

An important issue in the MSG algorithm is choosing the proper parameter values. There is no guaranteed method for identifying parameter values in the MSG methodology. However, it is very easy to find an upper bound for the QKP. Since the original objective function of the QKP is maximization, after converting the objective function into minimization form, zero will be a good upper bound for the problem. As the running times for the problems are not so long, trying to solve the QKP model with any positive upper bound is not difficult and a good upper bound can be found in a reasonable time. According to the algorithm, a vector $\left(u_{1}, c_{1}\right) \in R^{n} \times R_{+}$should be chosen at the initialization step. Zero vectors were used for all test problems as the initial

\footnotetext{
${ }^{1}$ http: //cedric. cnam.fr/ soutif/QKP/.
} 
value of the dual variables and each test problem was solved five times with different parameter $(\bar{H}, \bar{c}, \alpha, \delta)$ combinations. Ranges of the parameters were determined like that: $\bar{H} \geqslant 0, \bar{c} \in[2 c, 5 c], \alpha \in[2,10], \delta \in(0,2)$. Then, the best objective function value among five tests and its running time were reported as the result of the MSG algorithm.

Computational results for twenty QKP instances are illustrated in Table 1 as Group1, and Group 2. The left part of the table summarizes the properties of instances such as number of items $(n)$, density $(d)$ and the optimum value for each instance. The second and third parts contain solution results obtained by using GAMS MINLP solvers and the MSG algorithm with $s_{k}^{1}$ step size parameter. In order to solve the augmented Lagrangean function, CONOPT solver is used due to obtaining good solution results by using $s_{k}^{1}$ and CONOPT together. For each solution technique, the best objective function value among 5 experiments, closeness to the optimality in percentage $\left(E \%=100^{*}\right.$ (opt. valuevalue)/opt. value), and the elapsed time in second are given in Table 1. Better solutions are written in bold and underlined for each test instance.

Table 1

Performance of the MSG algorithm with step size parameter $s_{k}^{1}$ and CONOPT solver

\begin{tabular}{|c|c|c|c|c|c|c|c|c|c|c|c|c|}
\hline \multirow{2}{*}{\multicolumn{2}{|c|}{$\begin{array}{l}\text { Instances } \\
\text { Group 1 } \\
(n=100, d=0.25)\end{array}$}} & \multicolumn{8}{|c|}{ GAMS MINLP SOLVERS } & \multicolumn{3}{|c|}{ MSG } \\
\hline & & \multicolumn{2}{|c|}{ DICOPT } & \multicolumn{3}{|c|}{ SBB } & \multicolumn{3}{|c|}{ BARON } & \multirow[b]{2}{*}{ Value } & \multirow[b]{2}{*}{$\mathrm{E} \%$} & \multirow[b]{2}{*}{ Time } \\
\hline Opt. value & Value & $\mathrm{E} \%$ & Time & Value & $\mathrm{E} \%$ & Time & Value & $\mathrm{E} \%$ & Time & & & \\
\hline 18558 & 18511 & 0.25 & 0.74 & 18485 & 0.39 & 0.82 & 18514 & 0.24 & 0.78 & $\underline{18558}$ & 0.00 & 1.33 \\
\hline 56525 & $\underline{56525}$ & 0.00 & 0.53 & 55717 & 1.43 & 1.62 & 55578 & 1.68 & 4.97 & $\overline{56452}$ & 0.13 & 0.71 \\
\hline 3752 & $\underline{3752}$ & 0.00 & 0.37 & 3717 & 0.93 & 1.31 & 3538 & 5.70 & 2.33 & 3717 & 0.93 & 1.08 \\
\hline 50382 & $\underline{50382}$ & 0.00 & 0.08 & $\underline{50382}$ & 0.00 & 0.13 & $\underline{50382}$ & 0.00 & 0.89 & $\underline{50382}$ & 0.00 & 0.55 \\
\hline 61494 & $\overline{61494}$ & 0.00 & 0.31 & $\overline{61213}$ & 0.46 & 0.36 & 60983 & 0.83 & 0.25 & $\overline{61494}$ & 0.00 & 0.72 \\
\hline 36360 & $\underline{36360}$ & 0.00 & 0.46 & 36137 & 0.61 & 0.63 & 36137 & 0.61 & 0.89 & 36155 & 0.56 & 1.84 \\
\hline 14657 & $\underline{14657}$ & 0.00 & 0.35 & 14072 & 3.99 & 1.65 & 14282 & 2.56 & 0.78 & 14439 & 1.49 & 0.78 \\
\hline 20452 & 20369 & 0.41 & 0.78 & 19932 & 2.54 & 1.27 & 19932 & 2.54 & 0.66 & $\underline{20452}$ & 0.00 & 2.55 \\
\hline 35438 & $\underline{35438}$ & 0.00 & 0.37 & $\underline{35438}$ & 0.00 & 0.66 & 34924 & 1.45 & 0.81 & $\overline{35325}$ & 0.32 & 0.7 \\
\hline 24930 & $\underline{24915}$ & 0.06 & 0.59 & 24503 & 1.71 & 1.07 & 24748 & 0.73 & 0.78 & 24861 & 0.28 & 1.16 \\
\hline \multicolumn{2}{|c|}{ mean } & 0.07 & 0.46 & & 1.21 & 0.95 & & 1.63 & 1.31 & & 0.37 & 1.14 \\
\hline \multicolumn{13}{|c|}{$\begin{array}{l}\text { Group 2 } \\
(n=200, d=1)\end{array}$} \\
\hline Opt. value & Value & $\mathrm{E} \%$ & Time & Value & $\mathrm{E} \%$ & Time & Value & $\mathrm{E} \%$ & Time & Value & $\mathrm{E} \%$ & Time \\
\hline 937149 & $\underline{937149}$ & 0.00 & 0.6 & 935515 & 0.17 & 12.4 & 935700 & 0.15 & 78.7 & 933049 & 0.44 & 29.1 \\
\hline 303058 & $\overline{303058}$ & 0.00 & 0.91 & 301788 & 0.42 & 12.4 & 301759 & 0.43 & 121.97 & 294360 & 2.87 & 8.91 \\
\hline 29367 & $\overline{29367}$ & 0.00 & 0.58 & 28867 & 1.70 & 20.3 & 28867 & 1.70 & 1014.84 & 29162 & 0.70 & 13.4 \\
\hline 100838 & $\underline{\underline{100838}}$ & 0.00 & 1.35 & 100466 & 0.37 & 16.3 & 100466 & 0.37 & 270.38 & 100466 & 0.37 & 7.23 \\
\hline 786635 & $\overline{786635}$ & 0.00 & 1.12 & 785450 & 0.15 & 3.08 & 785450 & 0.15 & 297.33 & 784480 & 0.27 & 5.25 \\
\hline 41171 & $\underline{41171}$ & 0.00 & 0.66 & 39934 & 3.00 & 20.8 & 39318 & 4.50 & 266.17 & $\underline{41171}$ & 0.00 & 5.23 \\
\hline 701094 & $\overline{701094}$ & 0.00 & 0.7 & 698701 & 0.34 & 4.23 & 698701 & 0.34 & 305.5 & $\overline{698004}$ & 0.44 & 12.6 \\
\hline 782443 & $\overline{782443}$ & 0.00 & 0.73 & 780184 & 0.29 & 4.66 & 779785 & 0.34 & 226.38 & 767217 & 1.95 & 9.78 \\
\hline 628992 & $\overline{628925}$ & 0.01 & 0.78 & 627121 & 0.30 & 5.34 & 619257 & 1.55 & 256.55 & 627121 & 0.30 & 8.18 \\
\hline 378442 & $\underline{378442}$ & 0.00 & 1.14 & 376613 & 0.48 & 10 & 376744 & 0.45 & 206.14 & 370075 & 2.21 & 12.6 \\
\hline \multicolumn{2}{|c|}{ mean } & 0.00 & 0.86 & & 0.72 & 10.95 & & 1.00 & 214.65 & & 0.95 & 11.23 \\
\hline
\end{tabular}


As can be seen in Table 1, the most successful solver is clearly DICOPT. DICOPT has reached optimal solution in 18 of the 20 test problems. Besides, other 2 instances' results are quite close to optimal solution value except the first test problem. The MSG algorithm found the optimum solutions in 5 test problems. SBB found the optimum solution in only 2 test problems and BARON found the optimum solution in only 1 test problem. Additionally, according to solution times, the most successful solver is again DICOPT. It can be said that the MSG is the second best algorithm, SBB is the third and BARON is the last one regarding E\% (percent closeness to the optimal solution) and the solution time. On the other hand, it is strange that the MSG algorithm can find the optimal solution in the first test problem when DICOPT has got its worst performance. Indeed, the success of DICOPT is regarded surprising considering the optimal solutions found for all test problems with $n=200$. Nevertheless, it can be said that the MSG algorithm is as good an algorithm as other solvers in terms of solving QKP because it can solve all test problems in less than 1 second for Group 1. However, for Group 2, it is clear that the success of DICOPT is superior with respect to optimality and solution time.

In this study, in order to examine whether the success of the MSG can be increased, another step parameter $\left(s_{k}^{2}\right)$ and MINOS solver was used to solve these test instances. The obtained solutions are given in Table 2 .

As presented in Table 2, the MSG has obtained better solutions in 11 of the 20 instances. Two of them are in the first group (the seventh and ninth problems), and 9 of them are in the second group (all instances except the fifth one). The MSG can find the optimal solution for the seventh test instance of the first group. Besides, the obtained solution values were increased. It can therefore be suggested that better results are obtained by using step parameter $s_{k}^{2}$ and MINOS solver. On the other hand, the solution times are not as short as before. The mean percentage $E$ value is decreased from 0.37 to 0.2 in the first group test instances. This value is decreased from 0.95 to 0.23 for the second

Table 2

The performance of the MSG algorithm with step size parameter $s_{k}^{2}$ and MINOS solver

\begin{tabular}{cccccc}
\hline \multicolumn{3}{c}{ Instances } & \multicolumn{3}{c}{ MSG } \\
\hline $\mathrm{n}$ & $\mathrm{d}$ & Opt. value & Value & $\mathrm{E} \%$ & Time \\
\hline \multirow{2}{*}{100} & \multirow{2}{*}{0.25} & 14657 & $\mathbf{1 4 6 5 7}$ & 0.00 & 2.92 \\
& & 24930 & 24912 & 0.07 & 16.00 \\
200 & \multirow{2}{*}{1.00} & 937149 & 934467 & 0.29 & 63.30 \\
& & 303058 & 301965 & 0.36 & 87.30 \\
& & 29367 & 29176 & 0.65 & 184.08 \\
& & 100838 & 100837 & 0.00 & 143.96 \\
& & 786635 & 786018 & 0.08 & 73.81 \\
& & 701094 & 700260 & 0.12 & 95.02 \\
& & 782443 & 780148 & 0.29 & 64.87 \\
& 628992 & 628398 & 0.09 & 77.56 \\
& & 378442 & 377000 & 0.38 & 81.03 \\
\hline
\end{tabular}


group test instances results. On the other hand, the mean solution times were increased from $1.14 \mathrm{~s}$ to $2.84 \mathrm{~s}$ for the first group and from $11.23 \mathrm{~s}$ to $87.62 \mathrm{~s}$ for the second group. Despite this increase, the solution times are not so serious that it can not be tolerated, but finding better solution takes much time. These experiments show that the quality of the MSG solutions depends on choosing proper parameter values and choosing the step size parameter equation with solver. In other words, if these parameters are chosen properly, the MSG will be able to compete with other solvers.

\section{Conclusions}

In this study, it is shown that the MSG algorithm is a successful algorithm on solving QKP and it is as good as other GAMS MINLP solvers. Also, the MSG algorithm can find the optimum solution. However, the MSG algorithm fails to yield a similar great performance in solving the QAP. This result is considered to be surprising because the quadratic objective function of QKP is similar to that of QAP. The reason may be that DICOPT can handle a problem having one constraint like QKP easily. Nevertheless, solving performance of the MSG algorithm is too good to ignore. Besides, the performance of the QKP can be increased in terms of determining step parameters reasonably.

In further researches, the performance of the MSG algorithm may be tested on different integer programming models and different solvers or algorithms can be used to solve the sub problem.

\section{References}

Bazaraa, M.S., H.D. Sherali and C.M. Shetty (2006). Nonlinear Programming: Theory and Algorithms, 3rd ed. Wiley, New York.

Bertsekas, D.P. (1995). Nonlinear Programming, Athena Scientific, Belmont, Massachusetts.

Billionnet, A., and F. Calmels (1996). Linear programming for the 0-1 quadratic knapsack problem, European Journal of Operational Research, 92, 310-325.

Billionnet, A., and E. Soutif (2003). An exact method based on Lagrangean decomposition for the 0-1 quadratic knapsack problem, European Journal of Operational Research, 157(3), 565-575.

Bretthauer, K.M., and B. Shetty (1997). Quadratic resource allocation with generalized upper bounds. Operations Research Letters, 20, 51-57.

Burachik, R.S., and C.Y. Kaya (2007). An update rule and a convergence result for a penalty function method. Journal of Industrial Management and Optimization, 3(2), 381-398.

Burachik, R.S., R. Gasimov, N. Ismayilova and C.Y. Kaya (2006). On a modified subgradient algorithm for dual problems via sharp augmented Lagrangian, Journal of Global Optimization, 34(1), 55-78.

Burachik, R.S., C.Y. Kaya and M. Mammadov (in press). An inexact modified subgradient algorithm for nonconvex optimization, Computational Optimization and Applications. Published online: 29 Jan 2008, DOI: 10.1007/s10589-008-9168-7.

Caprara, A., D. Pisinger and P. Toth (1999). Exact solution of the quadratic knapsack problem. INFORMS Journal on Computing, 11, 125-137.

Chaillou, P., P. Hansen and Y. Mahieu (1986). Best network flow bound for the quadratic knapsack problem. Combinatorial Optimization of Lecture Notes in Mathematics, 1403, 225-235.

Gallo, G., P.L. Hammer and B. Simeone (1980). Quadratic knapsack problems. Mathematical Programming Study, 12, 132-149.

Gasimov, R. (2002). Augmented Lagrangean duality and nondifferantiable optimization methods in non-convex programming. Journal of Global Optimization, 24, 187-203. 
Gasimov, R.N., and O. Ustun (2005). Solving the quadratic assignment problems using modified subgradient algorithm. In M.B. Durmusoglu, C. Kahraman (Eds.). Proceedings of 35th International Conference on Computers \& Industrial Engineering, Istanbul, Turkey. pp. 757-762.

Gasimov, R.N., and A.M. Rubinov (2004). On augmented Lagrangeans for optimization problems with a single constraint. Journal of Global Optimization, 28(2), 153-173.

Gasimov, R.N., and O. Ustun (2007). Solving the quadratic assignment problem using F-MSG algorithm, Journal of Industrial and Management Optimization, 3(2), 173-191.

Helmberg, C., F. Rendl and R. Weismantel (2000). A semidefinite programming approach to the quadratic knapsack problem. Journal of Combinatorial Optimization, 4, 197-215.

Hunting, M., U. Faigle, W. Kern (2001). A Lagrangean relaxation approach to the edge-weighted clique problem, European Journal of Operational Research, 131, 119-131.

Julstrom, B.A. (2005). Greedy, genetic, and greedy genetic algorithms for the quadratic knapsack problem. In Proceedings of the Genetic and Evolutionary Computation Conference, Vol. 1. pp. 607-614.

Kellerer, H., U. Pferschy and D. Pisinger (2004). Knapsack Problems. Springer-Verlag, NY.

$\mathrm{Li}$, D. (1999). Zero duality gap in integer programming: P-norm surrogate constraint method. Operations Research Letters, 25, 89-96.

Li, H.L. (1992). An approximate method for local optima for nonlinear mixed integer programming problems. Computers and Operations Research, 19(5), 435-444.

Martin, W. (1998). Fast equi-partitioning of rectangular domains using stripe decomposition. Discrete Applied Mathematics, 82, 193-207.

Michelon, P., and N. Maculan (1993). Lagrangean methods for 0-1 quadratic problems. Discrete Applied Mathematics, 42, 257-269.

Michelon, P., and L. Veuilleux (1996). Lagrangean methods for the 0-1 quadratic knapsack problem. European Journal of Operational Research, 92, 326-341.

A. Sipahioglu is an assistant professor at Industrial Engineering Department, Osmangazi University, and he got his PhD degree from the same university, in 1996. His main research interests are mathematical modeling, integer programming and logistics management.

T. Saraç received $\mathrm{PhD}$ degree from the Eskisehir Osmangazi University, Turkey, 2007. She is a research assistant at the Industrial Engineering Department of the Eskisehir Osmangazi University. Her main research interests are operational research, integer programming, cutting stock problems, knapsack problems and genetic algorithms.

\section{Modifikuotas subgradientinis algoritmas 0-1 kuprinès uždaviniui spresti}

\section{Aydin SIPAHIOGLU, Tugba SARAÇ}

Šiame darbe tiriamas modifikuotas subgradientinis algoritmas (MSA) 0-1 kuprinės uždaviniui (QKU) spręsti. MSA buvo pasiūlytas R. Gasimovo dulioms problemoms spręsti pasinaudojus aštria papildyta Lagranžo funkcija. MSA pasižymi svarbiomis savybėmis. Pavyzdžiui, šis algoritmas konverguoja, garantuodamas nulinį dualumo skirtumą uždaviniuose su Lipšico tikslo ir ribojimu funkcijomis. Be to, MSA buvo sèkmingai pritaikytas neiškiliems tolydiniams ir kai kuriems kombinatoriniams uždaviniams spręsti. Šiame darbe MSA pritaikytas QKU su nelygybiniais ribojimais spręsti. Pirmiausia, sprendžiamas uždavinys yra suvedamas ị tolydaus iškilo optimizavimo uždavinị, pridejjus papildomų ribojimų. Po to konstruojamas dualusis uždavinys, ịvedus aštriają papildytą Lagranžo funkciją. Tuomet MSA yra pritaikomas dualiam uždaviniui spręsti, atsižvelgiant i lygybinius ribojimus normos skaičiavimui. Pasiūlytam metodui ịvertinti buvo išspręsti keli testiniai uždaviniai, paimti iš literatūros, pasinaudojus trimis skirtingomis MINLP procedūromis iš GAMS įrangos. 